ARTICLE

\title{
Carotenoids modulate kernel texture in maize by influencing amyloplast envelope integrity
}

Haihai Wang (1) 1,5, Yongcai Huang (1) 1,2, , Qiao Xiao ${ }^{1,2}$, Xing Huang1,2, Changsheng Li , Xiaoyan Gao', Qiong Wang ${ }^{1}$, Xiaoli Xiang ${ }^{1}$, Yidong Zhu ${ }^{1,2}$, Jiechen Wang ${ }^{1}$, Wenqin Wang $\mathbb{B}^{3}$, Brian A. Larkins ${ }^{4} \&$ Yongrui Wu (10) ${ }^{1 \times}$

The mechanism that creates vitreous endosperm in the mature maize kernel is poorly understood. We identified Vitreous endosperm 1 (Ven1) as a major QTL influencing this process. Ven1 encodes $\beta$-carotene hydroxylase 3 , an enzyme that modulates carotenoid composition in the amyloplast envelope. The A619 inbred contains a nonfunctional Ven1 allele, leading to a decrease in polar and an increase in non-polar carotenoids in the amyloplast. Coincidently, the stability of amyloplast membranes is increased during kernel desiccation. The lipid composition in endosperm cells in A619 is altered, giving rise to a persistent amyloplast envelope. These changes impede the gathering of protein bodies and prevent them from interacting with starch grains, creating air spaces that cause an opaque kernel phenotype. Genetic modifiers were identified that alter the effect of $V e n 7^{A 619}$, while maintaining a high $\beta$-carotene level. These studies provide insight for breeding vitreous kernel varieties and high vitamin $A$ content in maize.

\footnotetext{
${ }^{1}$ National Key Laboratory of Plant Molecular Genetics, CAS Center for Excellence in Molecular Plant Sciences, Shanghai Institute of Plant Physiology \& Ecology, Shanghai 200032, China. ${ }^{2}$ University of the Chinese Academy of Sciences, Beijing 100049, China. ${ }^{3}$ School of Agriculture and Biology, Shanghai Jiao Tong University, Shanghai 200240, China. ${ }^{4}$ School of Plant Sciences, University of Arizona, Tucson, Arizona 85721, USA. ${ }^{5}$ These authors contributed equally: Haihai Wang, Yongcai Huang. ${ }_{\text {email: yrwu@cemps.ac.cn }}$
} 
$\mathrm{M}$ aize kernel texture is a critical agronomic trait. It is influenced by the ratio of vitreous endosperm in the outer part of the kernel to the starchy endosperm in the center of the kernel ${ }^{1}$. Vitreous endosperm strengthens the kernel and protects the grains from mechanical damages during harvesting and shipping, whereas starchy endosperm is breakable and fragile, and is susceptible to pests and diseases ${ }^{2}$. Even though teosinte (wild maize) has a hard glume encasing the seed, it contains a large portion of vitreous endosperm. In this respect, there may have been evolutionary selection for vitreous endosperm in teosinte to enhance kernel integrity and passage through the gut of birds and other animals to aid seed dispersal. Maize grains containing a greater amount of vitreous endosperm have a higher kernel density and test weight and lower flotation indices $^{3,4}$. The vitreous region is also important for food processing, including making of grits and cornflakes, and other food products $^{5}$, and it is essential for making popcorn pop.

What creates vitreous endosperm is a longstanding question with many hypotheses, most of which are associated with synthesis of starch and storage proteins, and the factors affecting them. The major storage metabolites in the endosperm are starch and proteins, which account for 70 and $10 \%$, respectively, of the endosperm dry mass. Starch is synthesized and accumulated as starch grains (SGs) in amyloplasts. The major proteins are storage proteins, prolamins, collectively called zeins. Zeins contain a signal peptide that directs their synthesis into the lumen of the rough endoplasmic reticulum (RER), where they become organized into accretions called protein bodies $(\mathrm{PBs})^{6,7}$. During kernel maturation, SGs become surrounded by a proteinaceous matrix composed of PBs and amorphous cytoplasmic proteins. As the seed undergoes desiccation, the integrity of organellar membranes is lost and the peripheral endosperm cells, filled predominantly with PBs and SGs, form vitreous endosperm, while central endosperm cells, containing primarily SGs and few PBs, form the starchy endosperm ${ }^{1}$. Although a large number of mutations affecting zein proteins and starch synthesis have been identified and provided insight into the mechanisms that create vitreous endosperm $^{8,9}$, little is known about quantitative trait loci and their metabolic products that explain the genetic variation in kernel texture found in natural populations.

Maize inbred lines have great natural variation in kernel texture, ranging from nearly completely vitreous to completely opaque. W64A and A619 are typical yellow dent inbred lines that illustrate the natural variation for a normal amount and a small amount of vitreous endosperm. In this work, through backcrossing, we are able to identify and clone the gene responsible for a major QTL, Ven1, which affects kernel texture by regulating the $\beta$-carotene content. High levels of $\beta$-carotene appear to prevent the breakdown of amyloplast membranes and affect the quantity and composition of lipids in desiccating endosperm cells. This disrupts interactions between SGs and $\mathrm{PBs}$, leading to air spaces and, consequently, opacity of the mature endosperm.

\section{Results}

Ven1 is a principal QTL controlling maize kernel texture. Maize inbred lines W64A and A619 display differences in endosperm vitreousness (Fig. 1a-d). When measured, W64A kernels contain $80 \%$ vitreous endosperm, whereas A619 kernels have only $20 \%$ (Fig. 1c, d). On a light box, light transmission was observed for W64A but not A619 kernels (Fig. 1b). Scanning electron microscopy (SEM) revealed that the vitreous region of W64A endosperm contained SGs embedded in a protein matrix, but this matrix was not apparent in A619, which had loosely compacted SGs (Fig. 1c).
We used A619 to make reciprocal crosses with W64A and two other vitreous inbred lines, D1051 and P25, and found that all the F1 hybrids were vitreous (Supplementary Fig. 1), indicating the major QTL (designated vitreous endosperm 1, Ven1) responsible for the opaque endosperm phenotype in A619 (Ven $1^{A 619}$ ) is recessive. Maize kernel texture is a complex trait and is affected by many genetic and non-genetic factors. To clone Ven 1 , we created populations by continuously introgressing the dominant Ven $1^{W 64 A}$ allele into the A619 background via backcrossing. Progeny from the first two rounds of backcrossing $\left(\mathrm{F}_{1} \mathrm{BC}_{1}\right.$ and $\mathrm{F}_{1} \mathrm{BC}_{2}$ ) did not show phenotypic segregation, but in the third generation $\left(\mathrm{F}_{1} \mathrm{BC}_{3}\right)$, kernels displaying vitreous, opaque and intermediate phenotypes were observed (Supplementary Fig. 2a, b). With more backcrossing, an equal segregation of vitreous and opaque kernels clearly occurred in $\mathrm{F}_{1} \mathrm{BC}_{5}$ (Supplementary Fig. 2c). We also introgressed the $V e n 1^{D 1051}$ and $V e n 1^{P 25}$ alleles into A619 and found typical Mendelian segregation appeared in $\mathrm{F}_{1} \mathrm{BC}_{4}$ (Supplementary Fig. 2d, e). These results indicate the penetrance of the $V e n 1^{A 619}$ allele is strongly influenced by the genetic background. $\mathrm{F}_{1} \mathrm{BC}_{7}$, seeds with vitreous and opaque phenotypes were propagated by self-pollination for two generations, yielding the homozygous nearly isogenic lines (NILs), NILW64A and NILA619. The NILs were nearly identical in other plant phenotypes but exhibited an apparent difference in kernel texture (Fig. 1a-d). Although NILW64A contained a slightly smaller amount of vitreous endosperm than W64A, it had significantly more than A619 and NILA619 (Fig. 1c, d), thereby creating stronger light transmission on a light box (Fig. 1b).

We pooled the vitreous and opaque seeds segregating in the $\mathrm{F}_{1} \mathrm{BC}_{5}$ population of the W64A by A619 cross (Supplementary Fig. 2f) and performed Bulked Segregant Analysis (BSA) sequencing. This revealed only one candidate region located on chromosome 10 (Fig. 1e). Using $2000 \mathrm{~F}_{1} \mathrm{BC}_{7}$ individuals, the QTL was narrowed down to a $0.2 \mathrm{Mb}$ region (Fig. 1f), where five genes were annotated based on the B73 genome. RNA sequencing (RNA-seq) analysis of 18-DAP (day after pollination) endosperms of W64A, A619, NILW64A and NILA619 revealed only Zm00001d026056 was differentially expressed in W64A and NILW64A, versus A619 and NILA619; the other four genes were expressed at a similar level or expressed at low levels in the endosperm of these lines (Fig. 1g). We amplified and sequenced the gene body and regions within $1.2 \mathrm{~kb}$ up- and downstream of the coding region and found the Ven $1^{A 619}$ allele contains a 1250 bp deletion in the $3^{\prime}$ end, resulting in truncation of the Ven $1^{A 619}$ protein (Supplementary Fig. 3). Using a specific primer pair flanking this deletion ${ }^{10}$, we verified that $V e n 1^{A 619}$ was also associated with the opaque phenotype in the $\mathrm{F}_{1} \mathrm{BC}_{4}$ populations created by A619 crosses with D1051 and P25 (Supplementary Fig. 4). Consistent with the transcript levels, the VEN1 protein was barely detectable in A619 and NILA619, compared with W64A and NILW64A (Fig. 1h), indicating Ven1 $1^{A 619}$ is a nonfunctional allele.

To confirm that this mutation is responsible for the opaque endosperm phenotype of A619, we used RNA interference (RNAi) technology to knock down Ven1 expression in KN5585 (Supplementary Fig. 5a), a vitreous inbred line wildly used in China for maize transformation. Twenty independent $T_{0}$ $V e n 1 R N A i$ plants were recovered and all segregated vitreous and opaque seeds when self-pollinated. The transcript and protein levels of Ven 1 in progeny bearing the $R N A i$ transgene were dramatically reduced compared with wildtype kernels, as exemplified in photographs of two events (Fig. 1i, j). A representative ear is shown in Fig. 1k and Supplementary 5b, where three quarters of the seeds inherited the RNA $i$ construct and exhibited the opaque endosperm phenotype (Supplementary Fig. 5b). Homozygous Ven1RNAi ears showing a fully opaque 

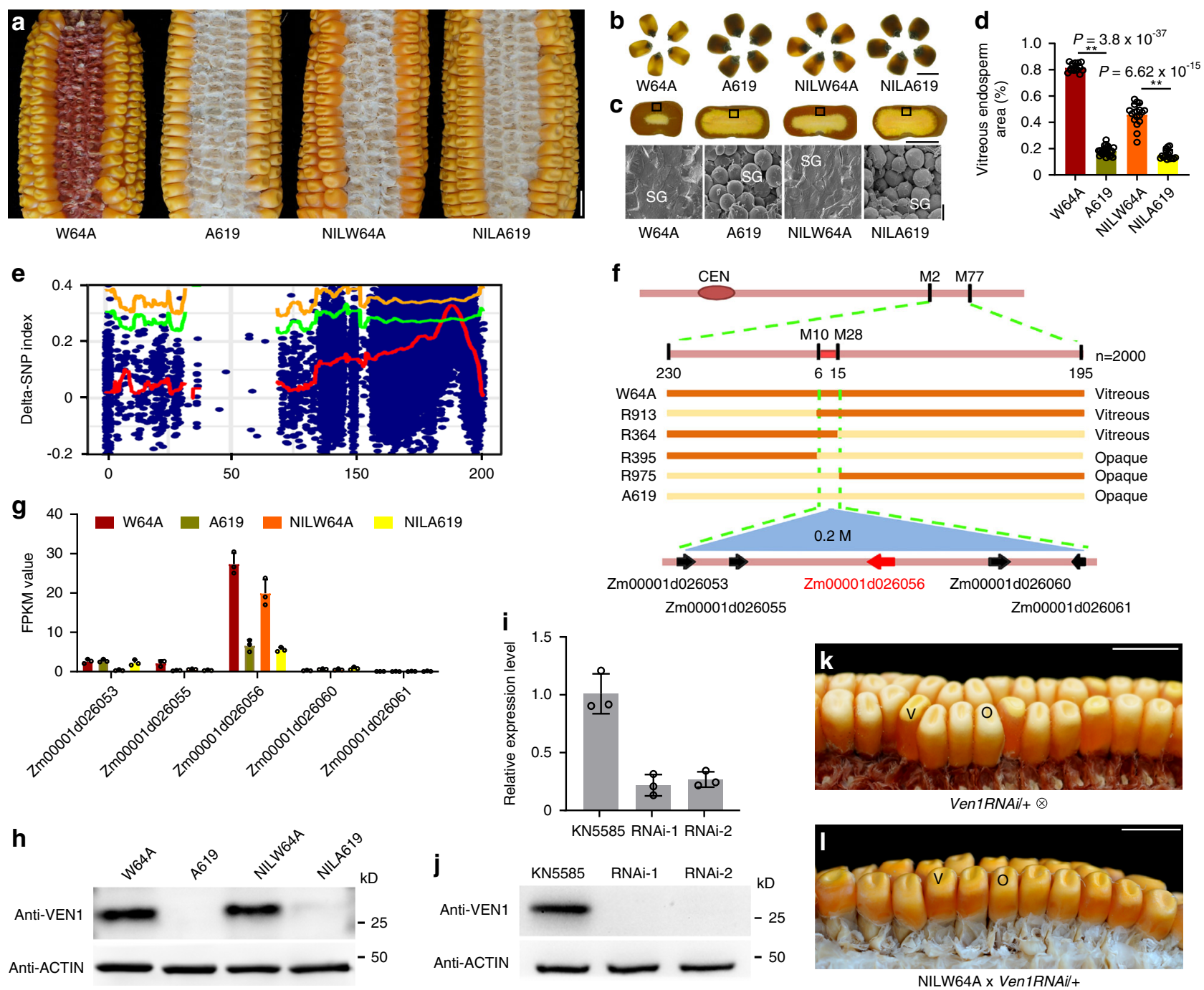

Fig. 1 Map-based cloning and genetic verification of Ven1. a Ear phenotypes of W64A, A619, NILW64A and NILA619. Scale bar, $1 \mathrm{~cm}$. b Kernel vitreousness as observed on the light box. W64A and NILW64A kernels are vitreous, and A619 and NILA619 opaque. Scale bar, $1 \mathrm{~cm}$. c Kernel transverse sections of W64A, A619, NILW64A and NILA619. Top panel, the sections observed under the natural light. Scale bar, $5 \mathrm{~mm}$; bottom panel, the areas boxed in the top observed by SEM. Scale bar, $10 \mu \mathrm{m}$. SG, starch grain. d Measurement of the vitreous endosperm area in kernel transverse sections. Data are presented as mean values $\pm \mathrm{SD}, n=15,23,18$ and 16 , respectively. ${ }^{\star *}$, significant differences at $P<0.01$ in two-sided Student's $t$-test. e Mapping-bysequencing of Ven1. Delta SNP index of two pools of vitreous and opaque kernels segregated from $\mathrm{F}_{1} \mathrm{BC}_{5}$ indicated a candidate QTL region in the long arm of chromosome 10. The red line is the mean value of SNP-index, green is the threshold line of $95 \%$ confidence level, and orange is $99 \%$ confidence level. f Fine mapping of Ven1. Ven1 was located in a $0.2 \mathrm{Mb}$ region between markers $\mathrm{M} 10$ and $\mathrm{M} 28$, containing five genes. $\mathbf{g}$ Expression level of the candidate genes in 18-DAP endosperms of two parents and two NILs. The values are shown as the means \pm sd of three biological replicates. $\mathbf{h}$ Immunoblotting analysis of VEN1 in seeds. ACTIN was used as an internal control. $\mathbf{i}$ qRT-PCR analysis of Ven1 in two independent transgenic lines. The values are shown as the means \pm s.d. of three biological replicates. Actin was used as an internal control. $\mathbf{j}$ Immunoblotting analysis of VEN1 in 18-DAP endosperms of two independent transgenic lines. ACTIN was used as an internal control. k Segregation of opaque kernels in a self-pollinated Ven1RNAi/+ ear. I Ear phenotype of NILW64A x Ven1RNAi/+. Vitreous and opaque kernels segregated equally. $\mathbf{k}, \mathbf{I}$ V, vitreous kernel; O, opaque kernel. Scale bar, $1 \mathrm{~cm}$. The source data underlying $\mathbf{d}, \mathbf{g}-\mathbf{j}$ are provided as a Source Data file.

seed set were obtained after propagating the transgenic seeds (Supplementary Fig. 5c). When NILW64A ears were fertilized by the pollen from heterozygous Ven1RNAi/+ plants, vitreous and opaque $F_{1}$ seeds segregated at a 1:1 ratio (Fig. 11, Supplementary Fig. 5d). These genetic data showed that Zm00001d026056 is the gene responsible for the opaque endosperm phenotype in A619.

Ven1 $1^{A 619}$ causes over-accumulation of nonpolar carotenoids. Quantitative RT-PCR revealed Ven1 is highly expressed in W64A endosperm, where RNA transcript levels gradually increased after 12 DAP, reaching a peak at 35 DAP (Fig. 2a). RNA in-situ hybridization showed Ven1 was predominantly expressed in starchy endosperm cells from the crown to the kernel center (Fig. 2b). To determine its subcellular localization, the VEN1 protein was fused to GFP and the resulting construct transiently expressed in Nicotiana benthamiana leaf cells. The signal from VEN1-GFP was observed to be localized to chloroplasts (Fig. 2c). Specifically, immunohistochemical assays clearly showed the distribution of VEN1 is restricted inside the amyloplast envelope in endosperm cells (Fig. 2d-f).

Ven1 encodes $\beta$-carotene hydroxylase 3 (HYD3), which hydroxylates the $\beta$-ionone ring of $\alpha$-/ $\beta$-carotene, producing xanthophylls (e.g. lutein and zeaxanthin) ${ }^{11}$. We measured the 

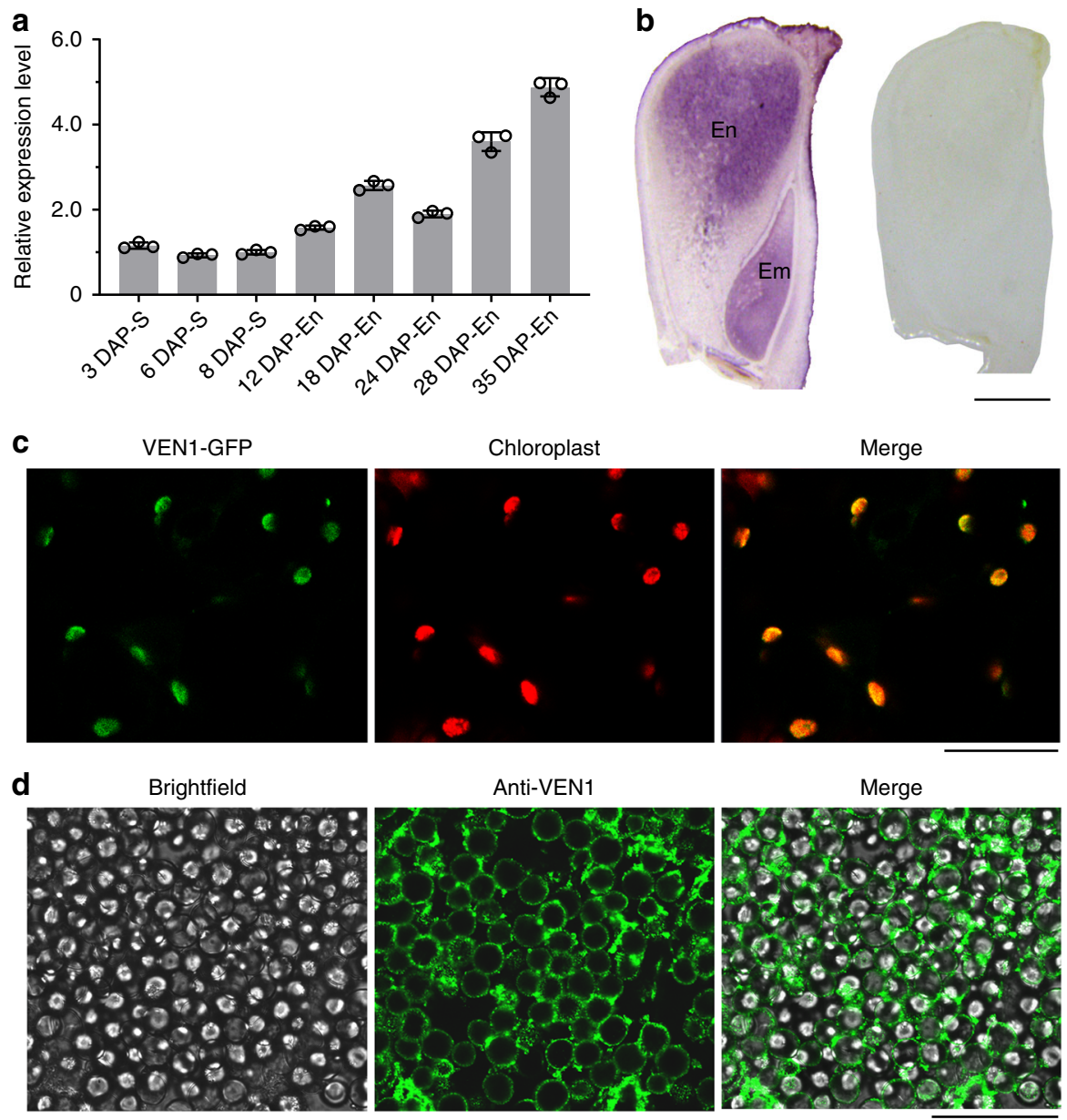

e

Brightfield

Anti-VEN1
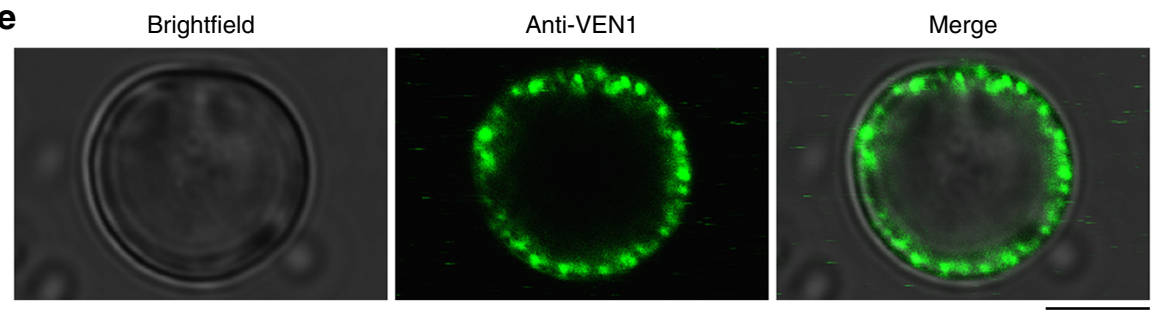

$\mathbf{f}$

Anti-VEN1
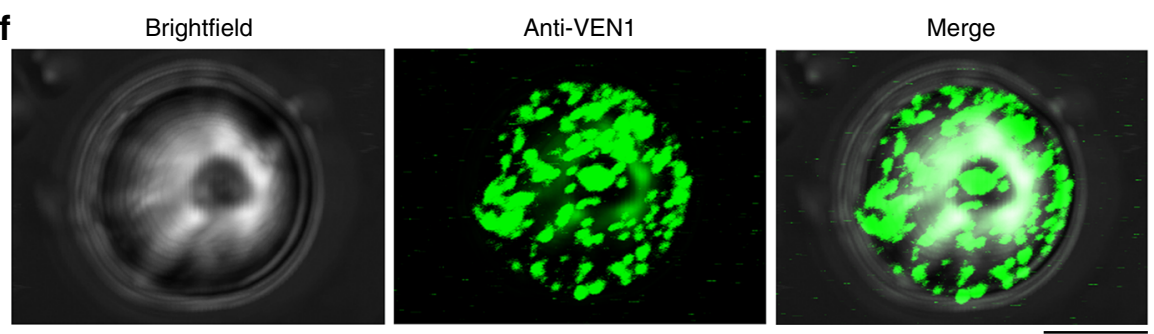

Fig. 2 The transcript and protein expression pattern of Ven1 during seed development. a RT-qPCR analysis of Ven1 expression during seed development. $\mathrm{S}$, Seeds; En, Endosperm. Data are presented as mean values $\pm S D, n=3$ biologically independent samples. b RNA in situ hybridization of Ven1 in the 18 DAP seed. Left panel, hybridization with antisense probes; right panel, hybridization with sense probes. Scale bar, 1 mm c Subcellular localization of VEN1GFP in Nicotiana benthamiana chloroplasts. Scale bar, $20 \mu \mathrm{m}$. d Localization of VEN1 in endosperm starchy cells by immunofluorescence. Scale bar, $50 \mu \mathrm{m}$. e Ringlike localization of VEN1 on a planar SG. Scale bar, $5 \mu \mathrm{m}$. $\mathbf{f}$ Localization of the VEN1 protein on the surface of a three-dimensional SG by Z-stack imaging. Scale bar, $5 \mu \mathrm{m}$. The source data underlying a are provided as a Source Data file.

total carotenoid content in 24- and 35-DAP endosperms and found it was not apparently different between NILW64A and NILA619 (Table 1). However, we found that the nonpolar carotenoids such as phytoene, lycopene, $\alpha$-carotene and $\beta$ carotene, molecules produced upstream of HYD3, accumulated at a significantly higher level in NILA619 than NILW64A, whereas the levels of the polar carotenoids, like lutein and zeaxanthin, downstream of HYD3, were the inverse (Table 1).

Ven1 $1^{A 619}$ influences SG-PB interactions. To investigate the mechanisms by which $V e n 1^{A 619}$ affects vitreous endosperm 
formation, we first examined zein protein synthesis. We found that A619 accumulates zein proteins at a level that is apparently lower than in W64A (Supplementary Fig. 6a). Therefore, it was possible that insufficient accumulation of zein proteins resulted in a reduction of $\mathrm{PB}$ number and $\mathrm{siz}^{5}$, which in turn caused an opaque phenotype in A619. However, zein RNA transcript and protein levels were not measurably different between NILW64A and NILA619 at all examined stages of grain filling (Supplementary Fig. 6b-e). Since protein content is usually determined by the maternal genotype and endosperm vitreousness by the filial genotype $^{9,12}$, we investigated the correlation of the two traits by pollinating A619 plants with a mixture of W64A and A619 pollen. We found the vitreous and opaque seeds (indicative of the cross with W64A and self-pollination of A619, respectively; Supplementary Fig. 6f) accumulated nearly identical levels of zein proteins (Supplementary Fig. 6g). These results demonstrated that the zein protein level itself is not associated with endosperm texture in this study. We then examined starch synthesis in NILW64A and NILA619 and found the expression of all starch synthetic genes and the total starch and amylose content showed no apparent difference in the two NILs (Supplementary Fig. 7a-d).

Because Ven 1 seems to have no direct effect on the zein proteins and starch synthesis (Supplementary Figs. 6 and 7), we explored how Ven $1^{A 619}$ affects PB and SG development, and subsequently their interaction (Supplementary Fig. 8). At 24 DAP, TEM revealed that $\mathrm{SGs}$ and $\mathrm{PBs}$ developed normally and showed no apparent difference in size and number between NILW64A and NILA619 (Supplementary Fig. 8a and c). This is consistent with the observation that starch and zein proteins were similarly accumulated in the NILs. When the endosperm cells were examined by SEM, we found that SGs in NILW64A were tightly surrounded by PBs, appearing to be embedded in a proteinaceous cytoskeletal matrix. By contrast, SGs in NILA619 were attached to noticeably fewer PBs and they exhibited an unequal distribution on the SG surface (Supplementary Fig. 8b, d). TEM of 30 DAP kernels revealed PBs in NILW64A endosperm cells increasingly gathered around SGs, whereas in NILA619 PBs were more randomly dispersed among SGs (Supplementary Fig. 8e, g). When observed by SEM, the SGs in NILW64A became embedded in a dense matrix of PBs and cytoplasmic contents. Probably due to tight compacting, the SGs began to form a polygonal shape. In NILA619, the majority of SGs were contacted by a small number of $\mathrm{PBs}$ and they maintained their spherical morphology (Supplementary Fig. 8f, h). Thirty five DAP is a critical time point for vitreous endosperm formation. By then most of the starchy endosperm cells have undergone programmed cell death and storage metabolite synthesis is slowing down, water content is rapidly decreasing, and the endosperm is entering the dough stage. In NILW64A, TEM showed that all the PBs converged together, creating a continuous network of matrix grids around the SGs; these grids, with SGs capsuled inside, appeared to function as the basic unit of vitreous endosperm formation. One could imagine that the later-formed vitreous endosperm is composed of numerous interconnecting units like this. In contrast, this grid structure was not organized in NILA619 (Supplementary Fig. 8i, k). In SEM, the SGs in NILW64A were observed to be tightly seamed together, with PBs developing distinctive features of vitreous endosperm formation, whereas the SGs in NILA619 were loosely packed with air space between them, a typical characteristic of opaque endosperm (Supplementary Fig. 8j, 1). These results demonstrated that compaction of PBs and SGs is affected during desiccation of NILA619 endosperm.

Ven1 $1^{A 619}$ affects the integrity of amyloplast membranes and lipid composition. To investigate the process of SG and PB interaction during desiccation of NIL-Ven $1^{A 619}$, we examined the 

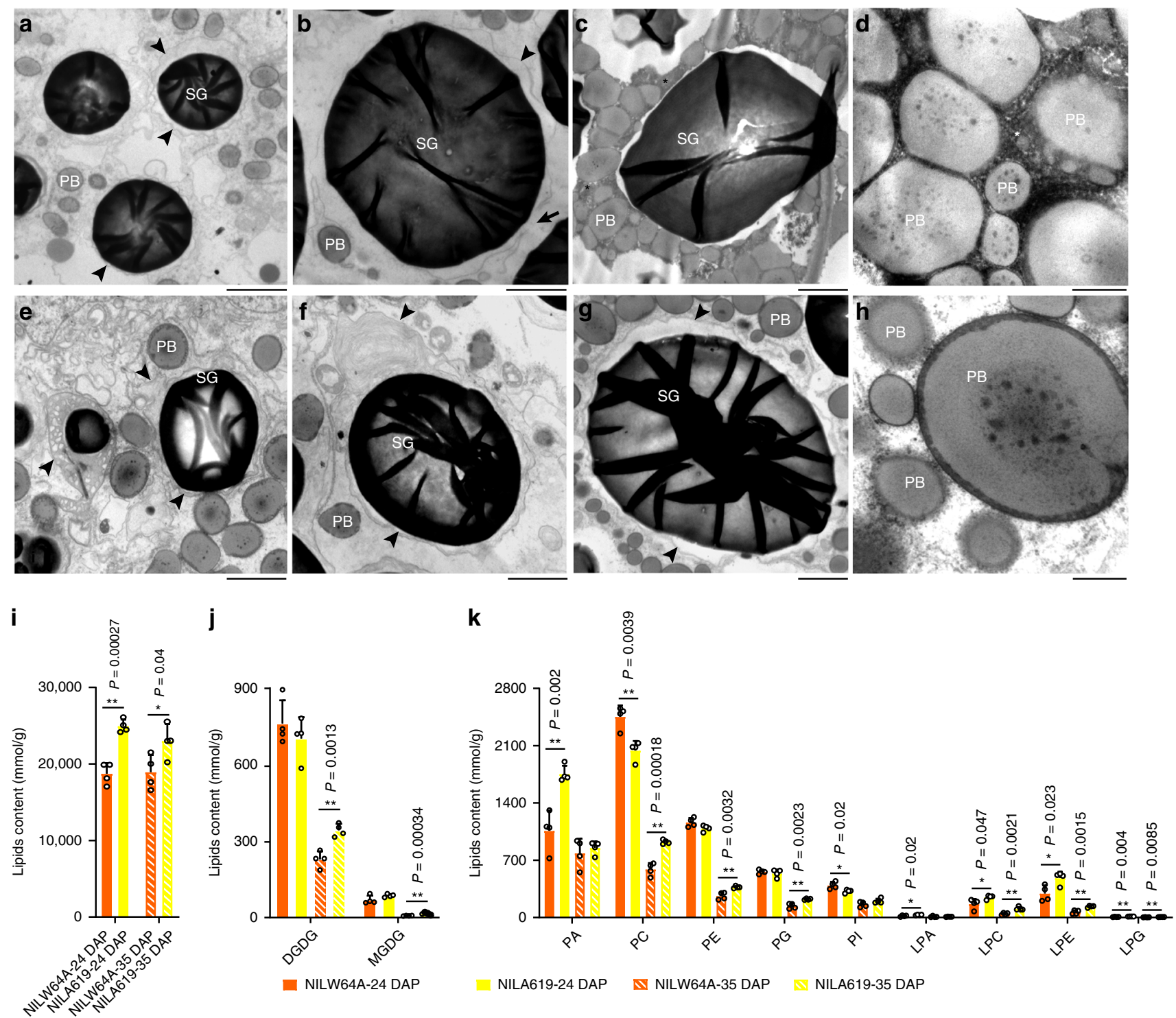

NILW64A-24 DAP

NILA619-24 DAP

\NILW64A-35 DAP

NILA619-35 DAP

Fig. 3 Stability of PB membrane and the changes in lipid composition in endosperm of NILs. a NILW64A endosperm cell at 18 DAP. b NILW64A endosperm cell at 24 DAP. c NILW64A endosperm cell at 35 DAP. d Tight packing of PBs and liposome-like structures in NILW64A at 35 DAP. e NILA619 endosperm cell at 18 DAP. f NILA619 endosperm cell at 24 DAP. $\mathbf{g}$ NILA619 endosperm cell at 35 DAP. h, Loose packing of PBs in NILA619 at 35 DAP. SG, starch grain; PB, protein body. Arrowheads indicate the amyloplast membranes; The arrow indicates broken amyloplast membranes in NILW64A; * indicates liposome-like structures among neighboring PBs in $\mathbf{d}$ and $\mathbf{h}$. Scale bar in $\mathbf{a}-\mathbf{c}, \mathbf{e}-\mathbf{g}, 2 \mu \mathrm{m}$; scale bar in $\mathbf{d}$ and $\mathbf{h}, 500 \mathrm{~nm}$. $\mathbf{i}$ The total lipid content in endosperms of NILs at 24 and 35 DAP. Data are presented as mean values \pm SD, $n=4$ biological replicates. ${ }^{*}$ and ${ }^{\star *}$, significant differences at $P<0.05$ and $P<0.01$ in two-sided Student's $t$-test, respectively. $\mathbf{j}-\mathbf{k}$, The content of galactolipids $\mathbf{j}$ and phospholipids $\mathbf{k}$ in endosperms of NILs at 24 and 35 DAP. Data are presented as mean values \pm SD, $n=4$ biological replicates. ${ }^{*}$ and ${ }^{*}$, significant differences at $P<0.05$ and $P<0.01$ in two-sided Student's $t$-test, respectively. DGDG, digalactosyldiacylglycerols; LPA, lyso-PA; LPC, lyso-PC; LPE, lyso-PE; LPG, lyso-PG; MGDG, monogalactosyldiacylglycerols, PA, phosphatidic acids; PC, phosphatidylcholines; PE, Phosphatidylethanolamines; PG, phosphatidylglycerols; PI, phosphatidylinositols. The source data underlying i-k are provided as a Source Data file.

organelles by TEM at higher resolution. Amyloplasts are a specialized plastid for starch synthesis and are bounded by two lipoprotein membranes. At 18 DAP, when the SGs in NILW64A were undergoing rapid development, we observed that the amyloplast membranes were intact, indicating they are functional for starch synthesis (Fig. 3a). At 24 DAP, when many SGs in NILW64A were reaching maturity, the amyloplast membranes began to break down, as evidenced by debris in their periphery (Fig. 3b). Because SGs at this stage differed in maturity, those with membranes in integrity or complete degradation (in the latter, the debris was absent in their periphery) were also observed (Supplementary Fig. 9a). At 35 DAP, the amyloplast membranes in NILW64A completely disappeared, enabling PBs to physically interact with SGs (Fig. 3c). It appeared that PBs were pulled together by undefined cytoplasmic components, perhaps by liposome-like structures, through hydrophobic interactions (Fig. 3d). In contrast, the amyloplast membranes in NILA619 behaved differently. At $18 \mathrm{DAP}$, the membranes appeared to be irregularly expanded, leading to formation of an amorphous structure (Fig. 3e). The membranes in NIL619 appeared to be more persistent; they were intact at 24 DAP (Fig. 3f) and remained intact at $35 \mathrm{DAP}$ (Fig. 3g). It appears the stability of the membranes creates a barrier that prevents PBs and the cytoplasmic matrix from approaching the SGs. There was little of the 
dense cytoplasmic contents around PBs compared to NILW64A (Fig. 3h). These results suggested Ven $1^{A 619}$ somehow influences the breakdown of amyloplast membranes, which is critical for allowing for SG-PB interaction.

Isolated amyloplast membranes contain about $70 \%$ galactolipid and $25 \%$ phospholipid (PL), but no neutral lipids like triacylglycerides (TAG) and fatty acids (FA) ${ }^{13}$. Monogalactosyldiacylglycerol (MGDG) and digalactosyldiacylglycerol (DGDG) provide both structural and functional organization of amyloplast. We measured the quantity and composition of lipids in the endosperm at 24 and 35 DAP and found they were markedly different between NILA619 and NILW64A (Fig. 3i-k; Supplementary Fig. 9b-e). In the endosperm, there are mainly neutral lipids, e.g., FA and TAG. In each NIL, total lipid content was not apparently altered at the two time points, but it was always significantly higher in NILA619 than NILW64A (Fig. 3i), suggesting an enhanced capacity for lipid synthesis. At 24 DAP, when amyloplast membranes of many SGs began to break down in NILW64A, only a few polar lipids (PA, LPC and LPE) accumulated at a mildly higher level in NILA619 than NILW64A (Fig. 3j, k). This suggested that although the breakdown of amyloplast membranes occurred in NILW64A, their main lipids, i.e. galactolipids were still present in endosperm cells. The apparent difference between the two NILs were the levels of FA and TAG (Supplementary Fig. 9c). By 35 DAP, when amyloplast membranes of most SGs completely degraded in NILW64A, the level of DGDG, which is a predominant galactolipid in amyloplast membranes, was significantly higher in NILA619 than NILW64A, as well as PL (PC, PE, PG) and lysoPL (LPC, LPE, LPG) (Fig. 3k, Supplementary Fig. 9b). It was evident that the contents of amyloplast lipids, MGDG, DGDG and other membrane phospholipids (PA, PC, PE and PG), were reduced at 35 DAP compared to those at 24 DAP in the two NILs. At this stage, FA and diacylglycerides (DAG, the minor neutral lipid constituent) were significantly higher in NILA619 than NILW64A, although TAG was not apparently different (Supplementary Fig. 9c-e). The difference in lipid composition between NILW64A and NILA619 is consistent with the differential status of amyloplast envelope integrity (Fig. 3b, c, f and g; Supplementary Fig. 9a).

Epistatic effect of carotenoid synthesis on the opaque phenotype in A619. To further investigate whether over-accumulation of non-polar carotenoids in A619 is associated with the opaque endosperm phenotype, we treated A619 with ethyl methanesulfonate (EMS) and screened for mutants with defects in carotenoid synthesis by observing endosperm color. We identified four single-gene recessive mutants displaying varying differences in endosperm color, suggesting these mutations occurred in different steps of carotenoid synthesis (Fig. 4a-e). A common feature of these mutants was the conversion of the opaque to a vitreous endosperm phenotype. Because these mutations function as suppressors of the Ven $1^{A 619}$ phenotype, we designated them vitreous endosperm suppressor 1, 2, 3-1 and 3-2 (ves1, ves2, ves3-1 and ves3-2, the last two were subsequently shown to be allelic mutations) (Fig. 4f-g). We measured the carotenoid composition and found the synthesis of lycopene, $\alpha$-carotene and $\beta$-carotene was suppressed in all these mutants (Fig. $4 \mathrm{~h}$ ), confirming a close relationship of the excessive accumulation of non-polar carotenoids and the opaque phenotype in A619 endosperm. We then cloned these genes by BSA sequencing (Supplementary Fig. 10a-d). The carotenoid biosynthesis pathway is conserved in maize and other plants, and the candidate genes, quantitative trait loci, and phenotypic loci are well characterized ${ }^{11,14-19}$. According to these reports, we summarized the maize carotenoid biosynthesis pathway and included the suppressors in Supplementary Fig. 10e. Ves1 encodes 4-hydroxyphenylpyruvate dioxygenase 1 (HPPD), which is the first committed step in synthesis of both plastoquinone and tocopherols in plants and functionally reverses the $p d s 1$ mutant phenotype in Arabidopsis ${ }^{20}$ (Supplementary Fig. 10e). The enzyme directly downstream of HPPD is homogentisate solanesyl transferase (HST), and its mutation in maize, $w 3$, showed blocked carotenoid synthesis by repressing the synthesis of PQ9, the oxidant required for phytoene desaturase (PDS). The $w 3$ mutant accumulates phytoene and exhibits a white kernel and seeding phenotype. Ves2 (also known as seed carotenoid deficient, $\mathrm{Scd}$ ) encodes a hydroxymethylbutenyl diphosphate synthase (HDS) converting 2C-methyl-D-erytrithol 2,4cyclodiphosphate to 1-hydroxy-2-methyl-2-(E)-butenyl 4diphosphate. Mutation of Scd inhibits the biosynthesis of MEPderived isoprenoids (carotenoids, chlorophylls, and tocopherols) and results in a white kennel, albino seedling and eventually lethal phenotype $^{21}$, similar to the ves 2 phenotypes. Ves 3 encodes the 1deoxy-D-xylulose-5-phosphate synthase (DXS), which is in upstream of VES2, a limiting enzyme for 2-C-methyl-D-erythritol 4-phosphate (MEP) for plant plastidic isoprenoid biosynthesis (Supplementary Fig. 10e) 22,23 . Sequencing revealed that ves1, 2 and 3-1 resulted from a premature stop codon in the second exon (Supplementary Fig. 10a-c) and ves3-2 was a missense mutation of DXS, in which Arg-210 was changed to Trp (Supplementary Fig. 10d). In contrast with the persistent amyloplast membranes in A619, TEM showed that the amyloplast membranes completely degraded in endosperm cells of ves 1, 2, 3-1 and 3-2 at 30 DAP, which allowed the PBs to converge around SGs and surround them (Fig. 4i). SEM revealed that the SGs in all suppressor mutant endosperms were tightly compacted with PBs and cytoplasmic contents (Fig. 4j). These results genetically supported that the upstream carotenoid biosynthetic pathway is epistatic to the Ven $1^{A 619}$ phenotype.

Screening Ven1 $1^{A 619}$ endosperm modifiers. During recurrent introgression of $V e n^{W 64 A}$ into A619, we did not observe segregation of the opaque phenotype until $\mathrm{F}_{1} \mathrm{BC}_{3}$ (Supplementary Fig. $2 \mathrm{a}-\mathrm{c}$ ). This was different from the cross of NILW64A and Ven1RNAi/+, where the $\mathrm{F}_{1}$ hybrids of W64A and Ven1RNAi/+ were vitreous (Supplementary Fig. 11), suggesting the presence of genetic modifiers in W64A that influence the opaque endosperm phenotype (caused by $V e n 1^{A 619}$ or Ven1RNAi). We analyzed the Ven 1 genotype in the 262 inbred lines (Supplementary Data 1) and found that 14 bear the Ven $1^{A 619}$ allele, of which 12 are vitreous and only two have an opaque endosperm in the mature kernel (Supplementary Fig. 12a). To identify Ven $1^{A 619}$ modifiers (Vem) in the natural population, we screened the 262 lines by crossing them with Ven1RNAi/+, no matter whether they are of the Ven $1^{A 619}$ genotype or not. If the inbred lacks modifiers, the $F_{1}$ progeny should segregate vitreous and opaque kernels at a $1: 1$ ratio; if does, all the progeny are expected to be vitreous. This screen resulted in identification of varying degrees of phenotypic modification in the natural population, wherein 182 were fully modified, 35 partially modified (showing a mosaic phenotype) and 45 totally unmodified (Supplementary Fig. 12a, b; Supplementary Data 1). Examples for each kind of modification are shown in Fig. 5a-c. These data demonstrated that a great proportion of germplasm $(30 \%)$ in the natural population are not suitable for provitamin A biofortification due to incomplete modification, while $V e n 1^{A 619}$ is a rare allele that has potential to increase $\beta$-carotene in maize grain.

A genome-wide association study (GWAS) for modifiers identified six obvious peaks $\left(P=5 \times 10^{-8}\right)$ distributed on chromosome 1, 3, 6 and 8 (Fig. 5d). Genetic variation and 


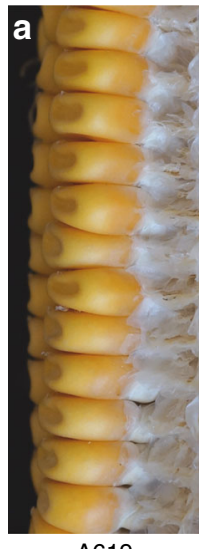

A619

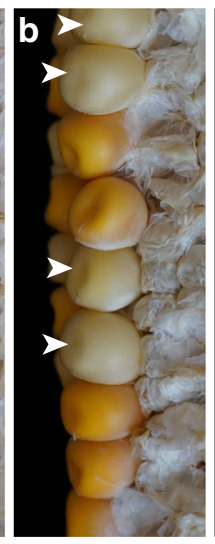

ves $1 /+\otimes$

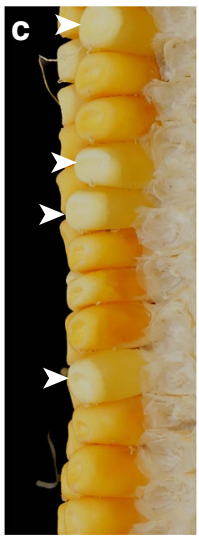

ves $2 /+\otimes$

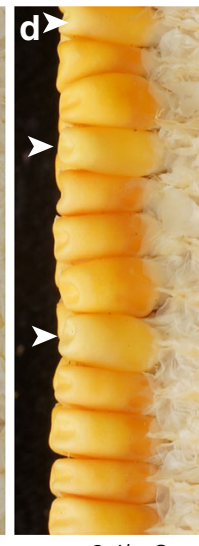

ves3-1/+ $\otimes$
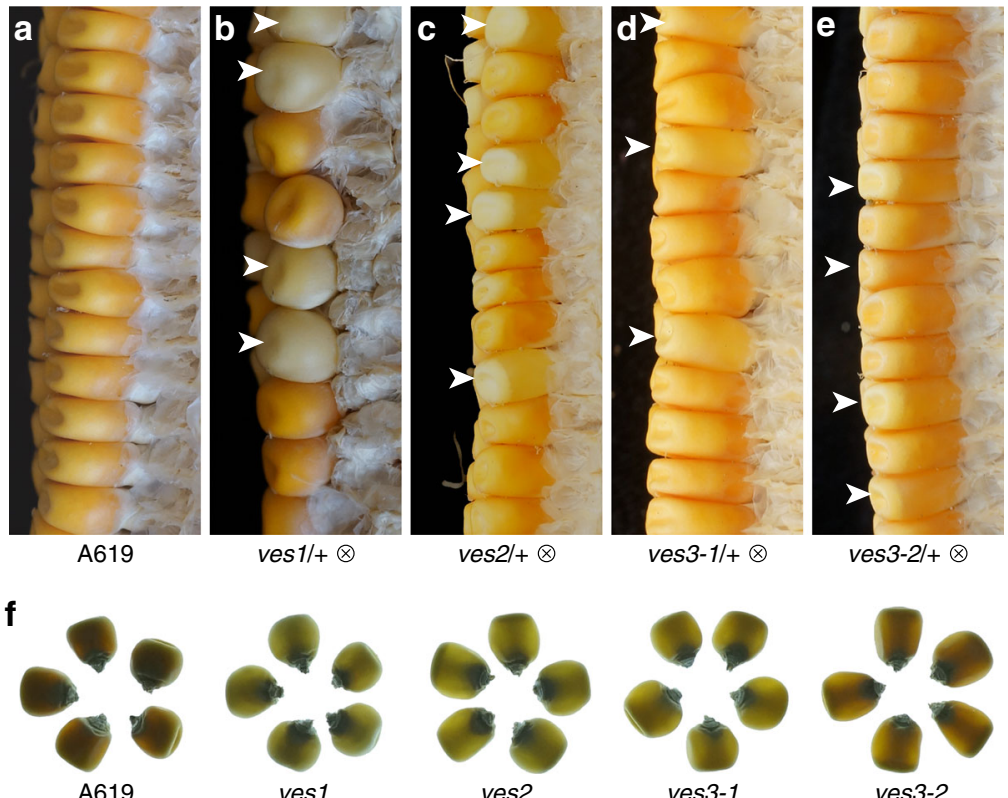

ves1

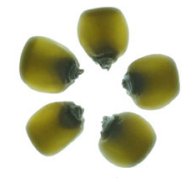

ves2
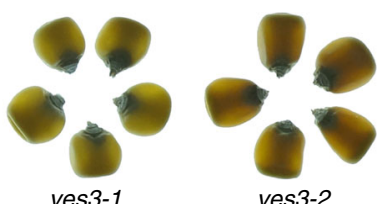

g

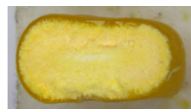

A619

i

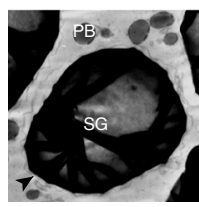

A619

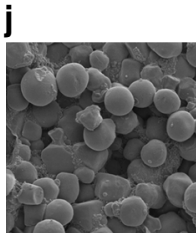

A619

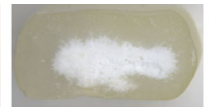

ves 1

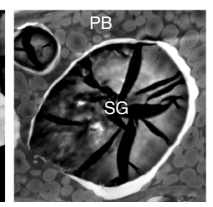

ves1

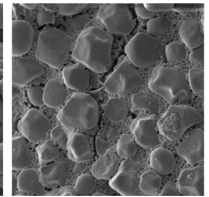

ves 1

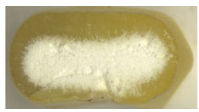

ves2

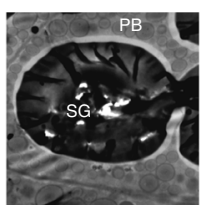

ves2

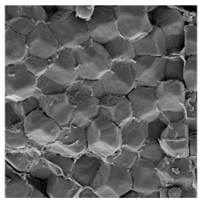

ves2

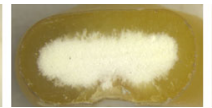

ves3-1

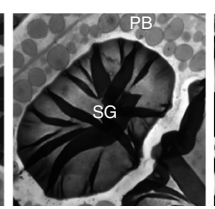

ves3-1

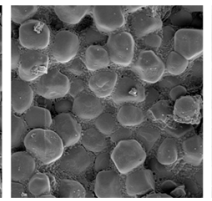

ves3-1

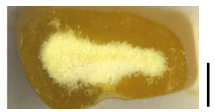

ves3-2

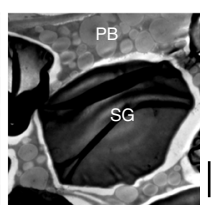

ves3-2

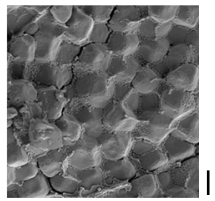

h
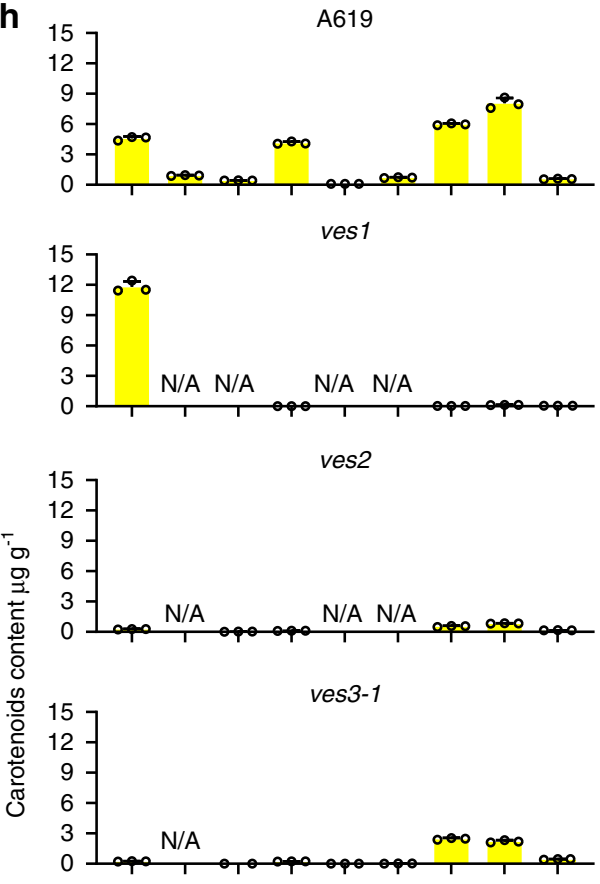

ves3-2
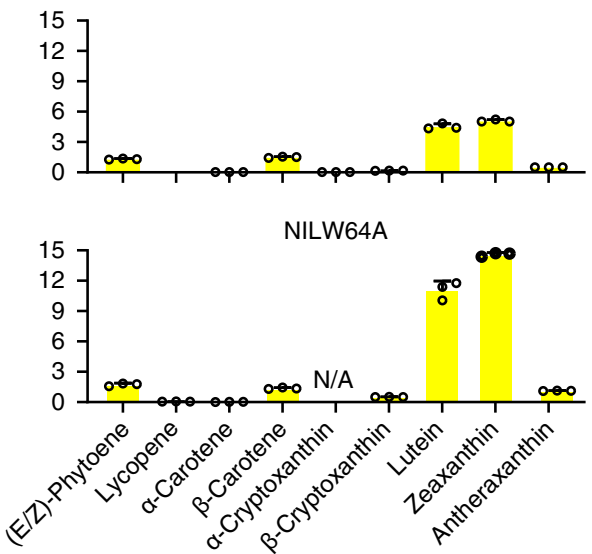

Fig. 4 Screening suppressors of Ven1A619. a A619 ear; b-e Ears segregating ves1 (b), ves2 (c), ves3-1 (d) and ves3-2 (e). Vitreous and carotenoid synthesis defective kernels are indicated with white arrowheads. Scale bar, $1 \mathrm{~cm}$. $\mathbf{f}$ Kernel vitreousness of A619 and the suppressors as observed on the light box. Scale bar, $1 \mathrm{~cm}$. $\mathbf{g}$ Kernel transverse sections of A619 and the suppressors. Scale bar, $5 \mathrm{~mm}$. $\mathbf{h}$ Altered carotenoid composition in endosperm tissues of A619, suppressors and NILW64A. Data are presented as mean values \pm SD, $n=3$ biological replicates. $\mathbf{i}$ TEM of the A619 and suppressors endosperm cells at 30 DAP. SG, starch grain; PB, protein body. Arrowheads indicate the amyloplast membranes. Scale bar, $2 \mu \mathrm{m}$. $\mathbf{j}$ SEM of the A619 and the suppressors endosperm cells at 30 DAP. Scale bar, $10 \mu \mathrm{m}$. The source data underlying $\mathbf{h}$ are provided as a Source Data file.

RNA-seq transcriptome analyses of W64A and A619 endosperms at 18 DAP revealed that nine genes in these regions were highly expressed in W64A, but lowly expressed in A619 (Supplementary Table 1). Among them, glutathione S-transferase 1 catalyzes conjugation of glutathione (GSH; $\gamma$-Glu-Cys-Gly) with electrophilic compounds, such as ROS and GSH, becoming an oxidized form, glutathione disulphide (GSSG) ${ }^{24}$. Therefore, higher expression of Gst 1 could alter the redox state in endosperm cells, which may in turn affect the stability of amyloplast membranes and SG$\mathrm{PB}$ interaction during vitreous endosperm formation.

\section{Discussion}

One of the first descriptions of cells in the vitreous endosperm was by Donald Duvick, who likened them to a box of white marbles (SGs) in which buckshot (PBs) was packed between the marbles and viscous cytoplasm functioned as a transparent glue between them, forming a structure like rigid concrete ${ }^{25}$. In this model, mutations affecting synthesis of SGs and zein PBs would affect the formation of vitreous endosperm ${ }^{9}$. Indeed, the starch mutants, waxy1 and amylose extender1, with a low- and high-amylose content in SGs, develop an opaque and nearly fully vitreous endosperm phenotype, respectively. Mutations either reducing zein protein synthesis or altering its deposition in PBs also create an opaque phenotype in the mature endosperm. Besides these mutations that directly affect starch and $\mathrm{PB}$ synthesis, there are many others that indirectly affect their synthesis and cause an opaque endosperm phenotype ${ }^{26-28}$. Evidence from these studies support the hypothesis that the number of PBs, the organization of zein proteins within them, and their interaction with SGs are essential factors for vitreous endosperm formation ${ }^{9}$. However, our understanding of the process by which vitreous endosperm forms 


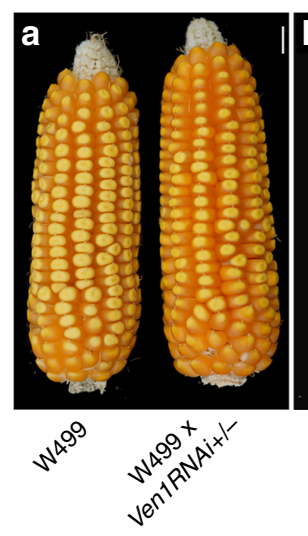

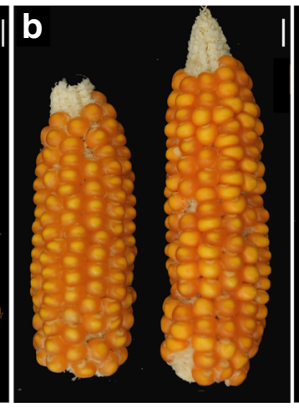

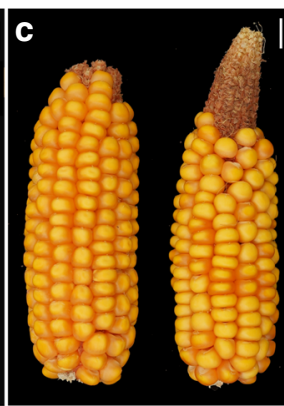

$1^{18}$

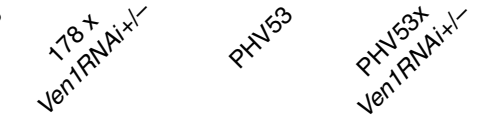

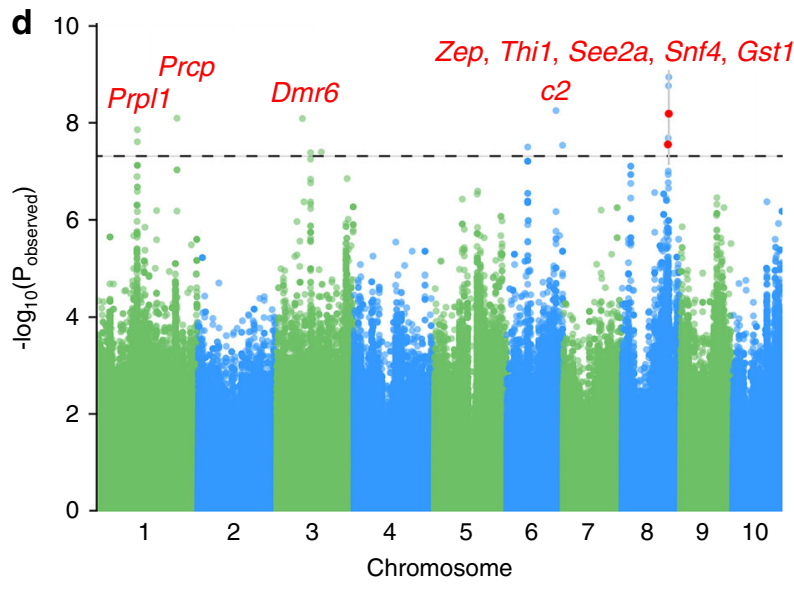

High

non-polar carotenoids

+ modifiers
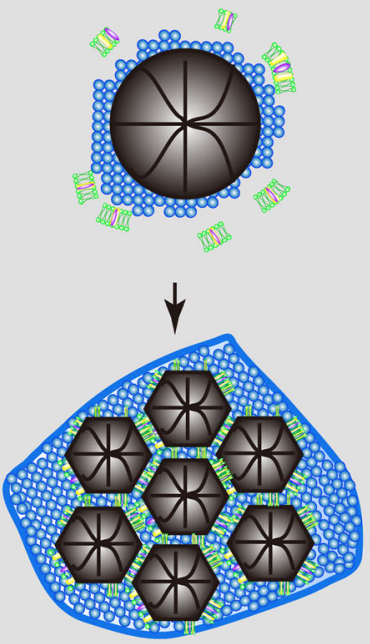

Vitreous endosperm
Modifiers

Non-polar carotenoids

Lipids

- PBs

Polygonal SGs

Alobular SGs

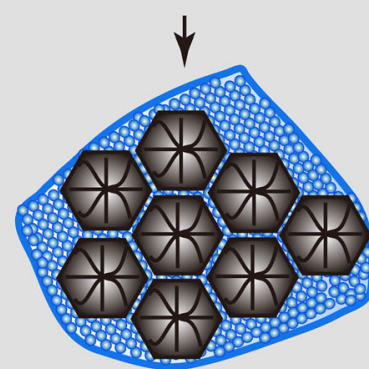

Vitreous endosperm
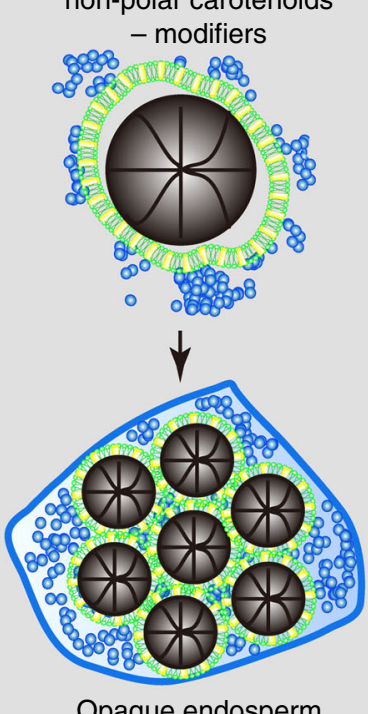

Opaque endosperm

Fig. 5 Screening Ven1 ${ }^{A 619}$ endosperm modifiers by GWAS and proposed model for vitreous endosperm formation. a-c Representative ear phenotypes showing full modification (a), partial modification (b) and totally unmodified (c) pollinated by Ven1RNAi/ +. d Manhattan plot of whether the endosperm contains Ven $7^{A 619}$ modifiers. The dashed line indicates the significance threshold of $P$-value $5 \times 10^{-8} .27$ unique SNPs are labelled with red dots, and the candidate genes are highlighted. The numbers on the horizontal axis indicate the maize chromosomes. e Proposed model for vitreous endosperm formation.

is incomplete, as other factors can influence it. For example, some mutations, like opaquel, have no apparent effect on zein or starch synthesis ${ }^{29}$. Indeed, the chemistry that creates the matrix between PBs and SGs during kernel desiccation is not understood. In this study, we demonstrated that Ven1, a QTL affecting the nature of carotenoids in the amyloplast and the stability of the amyloplast membrane, impacts interactions between SGs and PBs. An influence of the amyloplast membrane on vitreous endosperm formation was reported for the opaque 5 mutation ${ }^{30}$. Although the reason is unclear, this mutation affects mono-galactosyldiacylglcerol synthase, the enzyme responsible for synthesis of amyloplast membrane galactolipids. Whether or not this influences amyloplast membrane stability during kernel desiccation was not investigated in detail.

Vitreous endosperm begins to form at the periphery of the kernel by the late dough stage (35-40 DAP). This process could simply be a consequence of condensation of $\mathrm{PBs}$ and cellular contents onto SGs. At seed maturity, endomembrane systems would be expected to degrade as a result of PCD and water loss.
On the one hand, during vitreous endosperm formation the amyloplast envelope appears to break down before PB membranes (Fig. 3b, c). This would be critical to facilitate PBs and cytoplasmic contents condensing onto the SG surface and creating the matrix grid (Supplementary Fig. 8i, j; Fig. 3c). Our results support the hypothesis that the SG-PB interaction requires degradation of amyloplast membranes, which otherwise would form a barrier to this process (Supplementary Fig. 8k, l; Fig. 3f, g). On the other hand, PBs appear to stay in positions surrounding SGs around the time amyloplast membranes are about to degrade. Timing could be critical to achieve an optimal spatial arrangement of SGs and PBs, otherwise once the starchy endosperm tissue is dehydrated, PBs are unable to approach SGs and condense around them.

While the number and size of PBs matters for vitreous endosperm formation, the assembly of PBs is also a determining factor, as suggested by the opaque1 and floury $1^{31}$ mutations, which are associated with defects in synthesis of myosin or myosinrelated proteins, part of the cytoskeleton surrounding the RER ${ }^{32}$. 
Although the two NILs had identical zein protein abundance (Supplementary Fig. 6b), and thus a similar number and size of PBs (Supplementary Fig. 8a, c), it is evident that PBs don't gather around SGs (Supplementary Fig. 8c, g, k; Fig. 3e-g). A tentative explanation for the dispersal of PBs in NILA619 is the altered lipid content (Fig. 3i-k and Supplementary Fig. 9). Because zeins are a group of hydrophobic proteins, the association of PBs would involve hydrophobic interactions with lipids in the cytoplasm. In wheat, dark lipid inclusions (containing glycolipids, phospholipids, free fatty acids and monoglycerides) were found to organize in a liquid crystalline phase with protein matrix and SGs during vitreous endosperm formation ${ }^{33}$. The higher level of galactolipids (DGDG and MGDG) in NILA619 endosperms is associated with persistent amyloplast membranes at 35 DAP. In NILW64A, coincident with amyloplast membrane degradation, the membrane lipids significantly decreased (Fig. 3). As a result, the residual membrane lipids might organize the FA and DAG into liposome-like structures. Condensation of liposome-like structures between neighboring PBs is evident in NILW64A, but not NILA619 (Fig. 3c, d, g, h, indicated by asterisks), supporting this hypothesis. Vitreous endosperm formation might be related to the rearrangement of $\mathrm{PB}$ membranes and the lipid-mediated aggregation of $\mathrm{PBs}$ could be a prerequisite for the adhesion of PBs. As observed in durum wheat ${ }^{33}$, there might be a fusion between $\mathrm{PB}$ and amyloplast-derived membranes, especially during the dehydration phase, which will orientate the glass transition of the endosperm matrix towards a vitreous or an opaque solid state. Although maize endosperm contains a low level of lipids, many opaque and floury mutants exhibit defects in the structure of subcellular organelles, e.g., RER, $\mathrm{PBs}^{31}$ and amyloplasts $^{30}$, which are bounded by lipoprotein membranes. Alteration in lipid content might affect the PB-PB and SG-PB interactions (Fig. 3i, j), although further molecular and biochemical analyses are required to address this question.

Our data indicate that the composition of carotenoids in amyloplasts influence the stability of amyloplast membranes during kernel desiccation. Although there have been many studies of carotenoid interactions with membranes, little is known about the nature of carotenoids in amyloplast membranes. Ven $1^{A 619}$ is a non-functional allele of $H y d 3$, and results in an increased level of non-polar carotenoids in the kernel (Table 1). Since the VEN1 protein localizes to amyloplast membranes (Fig. $2 \mathrm{~d}-\mathrm{f}$ ), there are two possibilities that could explain the abnormalities and persistence of these membranes in mature endosperm of A619. First, VEN1 could function as a structural protein that negatively regulates the membrane stability. This is unlikely, however, because mutations in upstream genes affecting carotenoid synthesis also suppress the opaque kernel phenotype in the presence of Ven $1^{A 619}$ (Fig. 4). Although these mutations affect many other secondary metabolites such as tocopherol biosynthesis, apocarotenoid derivatives and other isoprenoids, they all have the common feature that the levels of lycopene, $\alpha$-carotene and $\beta$ carotene are concurrently reduced, indicating that upstream carotenoid biosynthetic genes are epistatic to the Ven $1^{A 619}$ phenotype and the altered carotenoid composition may trigger an unknown physicochemical or metabolic mechanism to protect amyloplast membranes from breaking down. Second, in contrast to polar carotenoids, e.g., lutein, zeaxamthin, and violaxanthin, that are primarily synthesized in amyloplast membranes in most inbred lines, non-polar carotenoids are in preponderance in NILA619. These carotenoids appear dispensable for the biological functions of amyloplasts, at least in terms of starch synthesis, which is supported by many high yielding commercial white maize varieties containing small amounts of carotenoids. Since the ves 1 endosperm accumulated only one kind of carotenoid, i.e. phytoene, and the level is obviously higher than that in the NILs and other suppressor lines (Fig. 4h), phytoene could be excluded and other nonpolar carotenoids (lycopene, $\alpha$-carotene or $\beta$-carotene) considered most likely responsible for the irregular and persistent amyloplast membranes. Moreover, carotenoids are precursors to hormones and many other apocarotenoids (including many new apocarotenoids recently discovered, such as $\beta$-cyclocitral derived from $\beta$-carotene, strigolactones, and others) ${ }^{18,34}$. The contents of these apocarotenoids may be changed in the endosperm of NILs and suppressors, which might also influence the stability of amyloplast membranes.

Since carotenoids are located in the membranes of the plastid envelop (here the non-photosynthetic amyloplast), it was logical to hypothesize a connection between the role of carotenoid composition and the physicochemical properties of amyloplast membranes. This hypothesis was strengthened by numerous works showing that, in vitro, carotenoids modulate the physical properties of model lipid membranes. For example, $\beta$-carotene tends to be randomly distributed within the hydrophobic interior of the bilayer envelope and increase the membrane fluidity, whereas polar carotenoids span lipid bilayer and have their polar groups anchored in the opposite polar zones of membrane; as a result, they increase the viscosity of the membrane ${ }^{35-37}$. In addition, zeaxanthin, but not $\beta$-carotene, increases the rigidity of model phospholipid membranes ${ }^{35,36}$. This effect of polar carotenoids is similar to the effect of cholesterol, i.e., limits the molecular motion of the lipid alkyl chains and favors the extended conformation of the alkyl chains of bilayer membranes. Probably, the elevated nonpolar carotenoids, particularly $\beta$-carotene, and decreased polar carotenoids, particularly zeaxanthin, created by $\operatorname{Ven} 1^{A 619}$ could increase the fluidity and hydrophobicity of amyloplast membranes and result in the irregularly expanded amyloplast membranes (Fig. 3e, f). The altered membrane physical properties could delay breakdown of amyloplast membranes in NILA619 endosperm, although the mechanism is unclear. As a result, NILA619 endosperm cells contain more amyloplast-specific lipids, DGDG, and phospholipids with a higher level of unsaturation than NILW64A (Fig. 3j-k and Supplementary Fig. 9b). Scavenging singlet oxygen $\left({ }^{1} \mathrm{O}_{2}\right)$ in membranes by $\beta$-carotene and its polar derivative, zeaxanthin, is effective in membranes containing unsaturated lipids (such as DGDG), but not in membranes containing saturated lipids ${ }^{38}$. One could envision that the higher level of unsaturated DGDG in NILA619 endosperm cells enhances the $\beta$-carotene-mediated antioxidant activity to protect the amyloplast membranes from oxidative damage and degradation during kernel dehydration.

$V e n 1^{A 619}$, previously identified as $c r t R B 1$ or $H y d 3$, has potential to be applied for high $\beta$-carotene breeding in corn ${ }^{10,11}$. Since knock-down of Ven1 expression causes an undesirable opaque kernel phenotype in many genetic backgrounds, use of the Ven $1^{A 619}$ allele or Ven1RNAi could be limited to germplasms with appropriate genetic modifiers (Supplementary Fig. 12). We created an effective screen for genetic modifiers in the natural population that can modify the opaque phenotype caused by Ven1RNAi (Fig. 5a). Indeed, several loci were identified, of which Gst1 encoding Glutathione S-transferase 1 was a major QTL (Fig. 5d), although their mechanisms remain to be understood.

Vitamin A deficiency (VAD) is the leading cause of preventable childhood blindness and is estimated to affect about one-third of the world's children under the age of five (information from the World Health Organization, https://www.who.int/nutrition/ topics/vad/en/). VAD usually results from dietary deficiency, as it mostly occurs in developing countries where the staple food is devoid of $\beta$-carotene. One economical approach to prevent VAD is to increase $\beta$-carotene levels in cereal grains. Maize, which is one of the most globally grown crops that provides nutritious food for humans, could serve as an ideal crop for provitamin A 
biofortification ${ }^{39}$. Although the level and composition of total carotenoids in natural maize populations varies dramatically, alleles that increase $\beta$-carotene in the grain are rare ${ }^{10}$, suggesting that, for an unknown reason, most selected varieties tend to contain a low $\beta$-carotene level. Because carotenoids are more concentrated in the vitreous endosperm than in the starchy endosperm, a better understanding of vitreous endosperm formation is potentially valuable for increasing $\beta$-carotene in maize grain.

In any case, maize kernel vitreousness is a complex phenotype and we presented new data that offers insight into how vitreous endosperm forms. Figure 5e shows a model illustrating one possible mechanism for interfering with the vitreous phenotype through carotenoids acting on amyloplast membranes. In white corn varieties or lines with a small amount of non-polar carotenoids, degradation of amyloplast membranes occurs coordinately with interaction between SGs and PBs as endosperm is entering the dough stage, creating the foundation for vitreous endosperm formation. These interactions can be disrupted by increased accumulation of non-polar carotenoids in amyloplast membranes, which stabilizes the membranes during endosperm desiccation by changing the membrane physical properties. In combination, these biochemical changes impair the condensation of PBs and cytoplasm contents onto SGs, leading to the formation of an opaque endosperm phenotype. This phenotype could hinder utilization of superior alleles that increase $\beta$-carotene content. Although the nature and mechanisms of genetic modifiers need to be further investigated, their presence in natural populations broadens the germplasm available for breeding high $\beta$-carotene varieties of corn that would benefit children suffering from vitamin A deficiencies.

\section{Methods}

Genetic materials. Yellow dent inbred lines W64A and A619 exhibiting vitreous and opaque endosperm phenotypes, respectively, were used for Ven1 mapping. By recurrent backcrossing, the Ven $1^{W 64 A}$ allele from W64A was introduced into the A619 background for genetic analysis. At $\mathrm{F}_{1} \mathrm{BC}_{5}$, the vitreous and opaque progeny seeds, segregating at a 1:1 ratio, were separated and divided into two pools for BSA sequencing. The vitreous and opaque seeds from $\mathrm{F}_{1} \mathrm{BC}_{7}$ were self-pollinated for two generations, yielding vitreous and opaque NILW64A and NILA619, respectively. Similar experiments were also conducted for two other vitreous inbred lines D1051 and P25 with A619.

Two hundred sixty two temperate maize inbred lines (Supplementary Data 1) provided by Dr. Jinsheng Lai at China Agricultural University, were used for the population genetic analysis.

To make the Ven1RNAi construct, reverse and forward Ven 1 cDNA fragments (400 bp in length) were amplified using two primer pairs: Ven1-SF2Xbal and Ven1-SR2Sac1, and Ven1-AFBspE1 and Ven1-ARBamH1. Expression of the Ven1RNAi cassette was driven by the $27-k D \gamma$-zein promoter. The construct was transformed into KN5585, a vitreous inbred line. Transgenic plants were confirmed by PCR using primers described elsewhere ${ }^{40}$.

Mapping Ven1. More than 100 vitreous and opaque kernels were selected for germination from the $\mathrm{F}_{1} \mathrm{BC}_{5}$ ears described above. Then high-quality genomic DNA was extracted individually at the seedling stage for subsequent sequencing. Libraries were constructed using the standard protocol of the Illumina TruSeq DNA PCR-free prep kit. Next-generation sequencing (NGS) technology was used to perform paired-end (PE) sequencing of the libraries based on the Illumina sequencing Nova Seq platform. The sequencing depth was $20 \mathrm{x}$ for the parents, and 50x for each mixed pool. Reads of the two bulks and two parents were filtered, quality evaluated, and sequence aligned to the B73 reference genome using bwa software. SNP calling was performed by GATK software and then calculated SNP index. Delta-SNP, the difference between the two bulks, was then determined and the statistical confidence intervals of index were calculated ${ }^{41}$. Sequencing service was provided by Personal Biotechnology Co., Ltd. Shanghai, China.

BSA analysis revealed a candidate interval of approximately $2 \mathrm{Mb}$. Polymorphic primers were designed (Supplementary Table 2) for fine mapping based on the sequencing data of the two parents. Using $2000 \mathrm{~F}_{1} \mathrm{BC}_{7}$ individuals, the Ven1 locus was located to an interval between two markers $137.1 \mathrm{M}$ and $137.3 \mathrm{M}$ on chromosome10. These two markers are genetically supported by 6 and 15 recombinant individuals, respectively. Recombinant individuals were selected and then self-pollinated. Further confirmation was by vitreous endosperm phenotypical identification from corresponding recombinants. These data support that Ven1 was mapped to the target interval segment. We also used these primers to test the $\mathrm{F}_{1} \mathrm{BC}_{4}$ populations created by $\mathrm{A} 619$ crosses with $\mathrm{D} 1051$ and $\mathrm{P} 25$, and found that the Ven1 markers were linked to the virtuous phenotype.

Phenotypic analysis. To examine kernel vitreousness, the mature, dry kernels were observed on a light box. To calculate the area of vitreous endosperm relative to the whole endosperm, the kernels were cut transversely and photographed under a light microscope stereoscope (Leica M165 FC). More than 15 seeds of each line were measured by ImageJ software. Then, the percentage of vitreous endosperm was calculated to determine the relative value.

SEM and TEM observation. For SEM observation, about $2 \mathrm{~mm}$ sections in the kernel's peripheral regions at 24,30 and 35 DAP were removed and immediately frozen in liquid nitrogen, and then dried using a freeze-vacuum dryer CoolSafe 554 PRO (ScanLaf). Sections from mature seeds were directly processed for SEM observation.

For TEM observation, about $1 \mathrm{~mm}$ sections in the kernel peripheral regions at 24,30 and 35 DAP were removed and immediately fixed in $2.5 \%$ glutaraldehyde in phosphate buffer $\mathrm{pH}$ 7.2, then dehydrated, and embedded following the standard protocol ${ }^{40}$. Ultrathin sections of the samples were cut with a diamond knife on a Leica EMUC6-FC6 ultramicrotome and imaged at $80 \mathrm{kV}$ with a Hitachi H-7650 transmission electron microscope.

Measurement of the proteins and starch. Twenty kernels selected from the middle of each ear had their embryo, seed coat and aleurone removed. The resulting endosperms were dried in an oven $\left(42{ }^{\circ} \mathrm{C}\right)$, and then ground into fine powder with a prototype machine $(60 \mathrm{HZ}, 60 \mathrm{~S})$. The powder was filtered through an 80 -mesh sieve to prepare samples for starch measurement.

For analysis of zeins in immature kernels, endosperms were collected at 18 and $24 \mathrm{DAP}$, and ground in the lipid nitrogen into fine powder. The extraction and analysis of zein proteins was according to the standard method of our laboratory 42 For assaying the total protein content, $60 \mathrm{mg}$ of endosperm powder was used for measurement by the instrument of ELEMENTAR Rapid N exceed. The starch content of endosperm was measured with a Total Starch Assay Kit (K-TSTA; Megazyme) following the standard protocol ${ }^{43}$. Amylose was quantified according to the Megazyme amylose/amylopectin assay procedure (K-AMYL; Megazyme). Six biological replicates were performed.

RNA-seq and RT-qPCR analysis. Developing endosperms were ground in liquid nitrogen. Total RNA was extracted by TRIzol reagent (Invitrogen, catalog number $15,596,018$ ) and then purified with an RNeasy Mini Kit (Qiagen, catalog number 74,106) after DNase 1 digestion (Qiagen, catalog number 79,254) following the manufacturer's protocol.

RNA-seq libraries of W64A, A619, NILW64A and NILA619 were prepared with an Illumina Standard library preparation kit and sequenced by HiSeqTM 2500 at OE Biotech Co, Ltd (Shanghai, China). Quality control checks on raw sequencing data were performed by FastQC combined with the NGS QC TOOLKIT v2.3.3. Clean reads were aligned to the B73 reference genome (RefGen_v3) and the reference gene model dataset (FGS 5b) using TopHat/ Bowtie2N (ccb.jhu.edu/software/tophat/). The gene expression value was calculated as fragments per kilobase of transcript per million mapped reads (FPKM). The FPKM value should be greater than zero for an expressed gene in the three biological replicates. Candidate genes and genes involved in synthesis of zeins and starch were selected and analyzed, respectively. The related web links are as follow: reference genome [ftp://ftp.ensemblgenomes.org/pub/plants/current/fasta/ zea_mays/dna/], reference transcript [ftp://ftp.ensemblgenomes.org/pub/plants/ current/fasta/zea_mays/cdna/], and annotations [ftp://ftp.ensemblgenomes.org/ pub/plants/current/fasta/zea_mays/cdna/].

For RT-qPCR analysis of Ven1 expression during seed development and filling, the digested RNA was used for reverse transcription with Super Script III First Strand Kit (Invitrogen). The resulting cDNA was diluted to $50 \mathrm{ng} / \mu \mathrm{L}$ for RT-qPCR with SYBR Green (TAKARA) on a CFX Connect Real-Time System (Bio Rad). The relative gene expression level was calculated using the comparative CT method ( ${ }_{\Delta} \mathrm{Ct}$ method), and the maize Actin gene was used as a control. The expression level in seeds at 8 DAP was set to 1 . In addition, expression of genes involved in zein and starch synthesis were also analyzed using 24 DAP and 30 DAP endosperms. Primers were listed in Supplementary Table 2.

Amplification of Ven1 in NILW64A and NILA619. Genomic DNA of NILW64A and NILA619 were extracted from seedling leaves using the CTAB method and used to amplify of the full-length genomic sequence of Ven1 using primers Ven1GF and Ven1-GR. The cDNAs reverse-transcribed from mRNAs of NILW64A and NILA619 endosperms were used for amplification of the Ven 1 coding sequence (CDS) with the primers Ven1-CF+Ven1W64A-CR, and Ven1-CF+Ven1A619-CR, respectively. The PCR products were then cloned into the pEASY Vector using pEASY-Blunt Zero Cloning Kit (TransGen, China), and six clones for each fragment were sequenced (TSINGKE Co., Ltd., China). 
RNA in situ hybridization. 18-DAP seeds from the middle region of the ears were harvested and fixed in $4 \%$ paraformaldehyde solution with $0.1 \%$ Triton X-100 and $0.1 \%$ Tween 20 in phosphate-buffered saline (PBS) (Takara, Cat\# T900) overnight. The seeds were dehydrated using a gradient concentration of ethanol and then were embedded in paraffin. A 400-bp cDNA fragment of Ven1 was amplified using the primer pair of Ven1-probeSac1 and Ven1-probeBamH1. The fragment was cloned into the pEASY vector for the synthesis of antisense and sense RNA probes according to the instructions for DIG RNA Labeling Mixture (Roche Cat\# 11175025910). $10-\mu \mathrm{m}$ seed sections were cut and then hybridized with the RNA probes at $50^{\circ} \mathrm{C}$ overnight. After blotting with Anti-digoxigenin AP-conjungate (Roche Cat\# 11093274910) and incubation with the NBT solution (Roche Cat\# 11383213001), the sections were observed and photographed with an ECLIPSE 80i microscope.

Antibody preparation. A fragment of the partial VEN1 protein from the $223^{\text {rd }}$ to $319^{\text {th }}$ amino acid, was used to produce antibodies by ABclonal, Wuhan, China. After affinity purification, the antibodies were used for Western blotting and immunofluorescence histochemistry.

Immunoblotting analysis. The nonzein protein extraction was according to the standard method of our laboratory ${ }^{42}$. Twenty $\mu$ g of nonzein proteins were used for immunoblotting analysis following the manufacture's protocol, (Bio-Rad, catalog number 162-0177). VEN1 and the control protein ACTIN antibodies were diluted $1: 1000$, while the secondary antibodies, goat anti-rabbit IgG-horseradish peroxidase (HRP) (Abmart, catalog number M21002L) and goat anti-mouse IgG-HRP (Abmart, catalog number M21001L), were diluted 1:5000 for performing the immune reaction. The membrane was treated with HRP chemiluminescent substrate reagent (Invitrogen, catalog number WP20005) and imaged using a Tanon5200 system (Tanon).

\section{Fluorescence immunohistochemistry analysis. Fluorescence immunohis-} tochemistry was used to detect the location of VEN1 in endosperm cells. Briefly, 18-DAP NILW64A endosperms were fixed, embedded and cut as described above for RNA in situ hybridization. 10- $\mu \mathrm{m}$ sections were blocked with $5 \%$ bovine serum albumin (BSA) (Yeasen Cat\# 36103ES60) in PBS for $1 \mathrm{~h}$ and then incubated with anti-VEN1 antibodies $(1: 100)$ overnight in the dark at $4{ }^{\circ} \mathrm{C}$. After washing three times with PBS, the samples were incubated with the secondary antibody, Alexa Fluor 488-conjugated goat anti-rabbit lgG (Yeasen Cat\# 33106ES60). The sections were observed with an LSM880 confocal microscope under Airyscan modes (Zeiss, Jena, Germany). Z-stack images of SGs were taken in the Airyscan superresolution mode.

Subcellular localization of VEN1 in Nicotiana benthamiana leaf cells. The fulllength Ven 1 coding sequence was amplified using primers Ven 1-KF and Ven1-BR and inserted into a pCAMBIA1301 plasmid driven by the $35 \mathrm{~S}$ promoter. The construct was transferred into Agrobacterium tumefaciens (GV3101 strain) and injected into 3-week-old Nicotiana benthamiana leaves. After two days infiltration, the transfected leaves were observed suing an LSM880 confocal microscope under Airyscan modes (Zeiss, Jena, Germany).

Determination of carotenoid composition. Carotenoid composition was measured by MetWare (Wuhan, China). To measure carotenoid composition, endosperms from three ears of NILW64A and NILA619 were collected and kept at -80 ${ }^{\circ} \mathrm{C}$ until use. The endosperms were homogenized and ground into fine powder, then dried using a freeze-vacuum dryer. Fifty $\mathrm{mg}$ of endosperm powder was extracted with mixture of $n$-hexane: acetone: ethanol $(2: 1: 1, V / V / V)$ and an internal standard was added. After two extractions, the supernatant was evaporated to dryness under nitrogen, and reconstituted in a solution of methanol: MTBE. The solution was passed through a $0.22 \mu \mathrm{m}$ filter and then analyzed with a LC-APCIMS/MS system (UHPLC, ExionLC ${ }^{m} \mathrm{AD}$, https://sciex.com.cn/; MS, Applied Biosystems 6500 Triple Quadrupole, https://sciex.com.cn/). A YMC C30 (3 um, $2 \mathrm{~mm} \times 100 \mathrm{~mm}$ ) column was used for HPLC analysis. Samples were eluted using a gradient from solvent $\mathrm{A}$, methanol: acetonitrile $(3: 1, \mathrm{~V} / \mathrm{V})$ added $0.01 \%$ BHT and $0.1 \%$ formic acid), to solvent $\mathrm{B}$, methyl tert-butyl ether $(0.01 \% \mathrm{BHT})$. The analysis was carried out at $28^{\circ} \mathrm{C}$ with a flow rate of $0.8 \mathrm{~mL} / \mathrm{min}$. MS analysis was performed using the API $6500 \mathrm{Q}$ TRAP LC/MS/MS System, equipped with an APCI Turbo Ion-Spray interface, operating in a positive ion mode and controlled by Analyst 1.6.3 software (AB Sciex). Carotenoid contents were detected by MetWare (http:// www.metware.cn/) based on the AB Sciex QTRAP6500 LC-MS/MS platform.

Determination of the lipid composition. Lipid composition was measured by MetWare (Wuhan, China) following the standard protocol ${ }^{44}$. For lipidomics analysis, $220 \mathrm{mg}$ dried endosperm powder was extracted with $3 \mathrm{~mL}$ of 2-propanol for $1 \mathrm{~h}$ and centrifuged $(3000 \mathrm{~g}, 5 \mathrm{~min})$. The pellet was extracted three times in dichloromethane: methanol $(2: 1, \mathrm{~V} / \mathrm{V})$ for $1 \mathrm{~h}$. The supernatant was pooled and dried on a rotary evaporator. Lipids were solubilized in a mixed solution of dichloromethane: methanol, to which $2 \mathrm{~mL}$ of $0.9 \% \mathrm{NaCl}$ in water was added. The lower organic phase was collected and dried under nitrogen flux. Then, lipids were solubilized in 2-propanol before UPLC analysis. Lipids were analyzed on an Ultra
High Performance Liquid Chromatography System (UPLC lc-30a) equipped with a Phenomenex Kinetex C18 column $(100 \times 2.1 \mathrm{~mm}, 2.6 \mu \mathrm{m})$ column. Lipids were eluted using a gradient from solvent $\mathrm{A}, \mathrm{H}_{2} \mathrm{O}: \mathrm{MeOH}$ : CAN (1:1:1 containing $5 \mathrm{mM}$ NH4Ac), to solvent B, IPA: ACN (5:1 containing $\left.5 \mathrm{mM} \mathrm{NH}_{4} \mathrm{Ac}\right)$. The analysis was carried out at $60^{\circ} \mathrm{C}$ with a flow rate of $0.4 \mathrm{~mL} / \mathrm{min}$. MS analysis was performed using the AB Sciex TripleTOF ${ }^{\circledR} 6600$ System, operating in a positive ion mode and controlled by Analyst 1.6.3 software (AB Sciex). Glycolipids were quantified using $\mathrm{PE}$ as the standard.

EMS mutagenesis and cloning of suppressors. A619 pollen was collected in a 50 $\mathrm{mL}$ tube, added to EMS reagent diluted in mineral oil (1:1000, vol/vol), and incubated for $45 \mathrm{~min}$. The mutagenized pollen was applied to the A619 ears. The resulting seeds were planted and surviving plants self-pollinated, yielding more than 2000 ears. Among them, four segregated kernels that were one quarter vitreous and defective in carotenoid synthesis. The four mutants were cloned by BSA sequencing. The BSA sequencing and analyses were provided by OE biotech Co., Ltd. (Shanghai, China).

\section{Screening genetic modifiers of Ven1RNAi in the natural population. Two} hundred sixty two inbred lines were pollinated by Ven1RNAi/+. The $\mathrm{F}_{1}$ endosperm phenotypes were divided into 3 levels: vitreous (completely modified), mosaic (partially modified) and opaque (completely unmodified). According to the phenotype, GAWS analysis was performed using the vitreous and opaque groups to screen the modifiers in the natural population following the standard protocol ${ }^{45}$.

Statistics and reproducibility. Three independent replications were performed to observe the vitreous endosperm formation in W64A, A616, NILW64A and NILA619 by SEM shown in Fig. 1c and at least six kernels were analyzed in each experiment. Western blotting experiments in Fig. $1 \mathrm{~h}$ and $\mathrm{j}$ were repeated three times. Confocal analyses of the HYD3 localization in leaf and endosperm cells were performed three independent times in Fig. 2c-f. TEM and SEM analyses in Figs. 3, 4 and Supplementary Fig. 8 were performed with 20 kernels from three cobs in each experiment. At least 100 endosperm cells were observed to investigate the changes in starch granule membranes shown in Figs. 3a-h, $4 \mathrm{i}$ and Supplementary Fig. 9a. 182 and 200 individual plants were used to perform genetic linkage analysis by PCR in two BC4F1 populations, respectively, shown in Supplementary Fig. $4 \mathrm{~b}$ and $\mathrm{d}$. Three independent replications were performed to analyze the zein protein content shown in Supplementary Fig. 6a, b, g.

Reporting summary. Further information on research design is available in the Nature Research Reporting Summary linked to this article.

\section{Data availability}

Data supporting the findings of this work are available within the paper and its Source Data. A reporting summary for this Article is available as a Supplementary Information file. The genetic materials generated and analyzed during the current study are available from the corresponding author upon request. Source data are provided with this paper.

Received: 5 June 2020; Accepted: 3 October 2020;

Published online: 22 October 2020

\section{References}

1. Gibbon, B. C. \& Larkins, B. A. Molecular genetic approaches to developing quality protein maize. Trends Genet. 21, 227-233 (2005)

2. Vasal S. K., Villegas E., Bjarnason M., Gelaw B., Goertz P. Genetic modifiers and breeding strategies in developing hard endosperm opaque-2 materials. In Improvement of Quality Traits of Maize for Grain and Silage Use (eds Pollmer, W. G. \& Phillips, R. H.). Martinus Nijhoff (1980).

3. Caballero-Rothar, N. N., Abdala, L. J., Borras, L. \& Gerde, J. A. Role of yield genetic progress on the biochemical determinants of maize kernel hardness. $J$ Cereal Sci. 87, 301-310 (2019).

4. Gayral, M. et al. Transition from vitreous to floury endosperm in maize (Zea mays L.) kernels is related to protein and starch gradients. J. Cereal Sci. 68, 148-154 (2016)

5. Fox, G. \& Manley, M. Hardness methods for testing maize kernels. J. Agric Food Chem. 57, 5647-5657 (2009).

6. Larkins, B. A. \& Hurkman, W. J. Synthesis and deposition of zein in protein bodies of maize endosperm. Plant Physiol. 62, 256-263 (1978).

7. Lending, C. R. \& Larkins, B. A. Changes in the zein composition of protein bodies during maize endosperm development. Plant cell 1, 1011-1023 (1989).

8. $\mathrm{Wu}, \mathrm{Y} .$, Messing, J. Understanding and improving protein traits in maize. In Achieving sustainable cultivation of maize $\mathrm{Vol} 1$ : From improved varieties to local applications (eds Watson, D.) (Burleigh Dodds Science Publishing, 2017). 
9. Larkins B. A., Wu Y., Song R., Messing J. Maize Seed Storage Proteins. In: Maize Kernel Development (ed. Larkins, B. A.) (CABI, 2017).

10. Yan, J. et al. Rare genetic variation at Zea mays crtRB1 increases beta-carotene in maize grain. Nat. Genet. 42, 322-327 (2010).

11. Vallabhaneni, R. et al. Metabolite sorting of a germplasm collection reveals the hydroxylase 3 locus as a new target for maize provitamin A biofortification. Plant Physiol. 151, 1635-1645 (2009).

12. $\mathrm{Wu}, \mathrm{Y}$. \& Messing, J. RNA interference can rebalance the nitrogen sink of maize seeds without losing hard endosperm. PLoS ONE 7, e32850 (2012).

13. Gardner, H. W., Miernyk, J. A., Christianson, D. D. \& Khoo, U. Isolation and characterization of an amyloplast envelope-enriched fraction from immature maize endosperm. Physiol. Plant 69, 541-549 (1987).

14. Li, F., Murillo, C. \& Wurtzel, E. T. Maize Y9 encodes a product essential for 15-cis-zeta-carotene isomerization. Plant Physiol. 144, 1181-1189 (2007).

15. Chen, Y., Li, F. \& Wurtzel, E. T. Isolation and characterization of the Z-ISO gene encoding a missing component of carotenoid biosynthesis in plants. Plant Physiol. 153, 66-79 (2010).

16. Quinlan, R. F. et al. Synergistic interactions between carotene ring hydroxylases drive lutein formation in plant carotenoid biosynthesis. Plant Physiol. 160, 204-214 (2012).

17. Wurtzel, E. T., Cuttriss, A. \& Vallabhaneni, R. Maize provitamin a carotenoids, current resources, and future metabolic engineering challenges. Front. Plant Sci. 3, 29 (2012).

18. Wurtzel, E. T. Changing form and function through carotenoids and synthetic biology. Plant Physiol. 179, 830-843 (2019).

19. Beltran, J. et al. Control of carotenoid biosynthesis through a heme-based cistrans isomerase. Nat. Chem. Biol. 11, 598-605 (2015).

20. Norris, S. R., Shen, X. \& DellaPenna, D. Complementation of the Arabidopsis pds1 mutation with the gene encoding p-hydroxyphenylpyruvate dioxygenase. Plant Physiol. 117, 1317-1323 (1998).

21. Zhang, L. et al. Seed carotenoid deficient functions in isoprenoid biosynthesis via the plastid MEP pathway. Plant Physiol. 179, 1723-1738 (2019).

22. Estevez, J. M., Cantero, A., Reindl, A., Reichler, S. \& Leon, P. 1-Deoxy-Dxylulose-5-phosphate synthase, a limiting enzyme for plastidic isoprenoid biosynthesis in plants. J. Biol. Chem. 276, 22901-22909 (2001).

23. Vallabhaneni, R. \& Wurtzel, E. T. Timing and biosynthetic potential for carotenoid accumulation in genetically diverse germplasm of maize. Plant Physiol. 150, 562-572 (2009).

24. Labrou, N. E., Papageorgiou, A. C., Pavli, O. \& Flemetakis, E. Plant GSTome: structure and functional role in xenome network and plant stress response. Curr. Opin. Biotechnol. 32, 186-194 (2015).

25. Duvick, D. N. Protein granules of maize endosperm cells. Cereal Chem. 38 , 374-385 (1961).

26. Miclaus, M., Wu, Y., Xu, J. H., Dooner, H. K. \& Messing, J. The maize highlysine mutant opaque7 is defective in an acyl-CoA synthetase-like protein. Genetics 189, 1271-1280 (2011).

27. Wang, G. et al. Opaque7 encodes an acyl-activating enzyme-like protein that affects storage protein synthesis in maize endosperm. Genetics 189, 1281-1295 (2011).

28. Yang, J., Fu, M., Ji, C., Huang, Y. \& Wu, Y. Maize oxalyl-CoA decarboxylase1 degrades oxalate and affects the seed metabolome and nutritional quality. Plant Cell 30, 2447-2462 (2018).

29. Wang, G. et al. Opaquel encodes a myosin XI motor protein that is required for endoplasmic reticulum motility and protein body formation in maize endosperm. Plant Cell 24, 3447-3462 (2012).

30. Myers, A. M. et al. Maize opaque5 encodes monogalactosyldiacylglycerol synthase and specifically affects galactolipids necessary for amyloplast and chloroplast function. Plant Cell 23, 2331-2347 (2011).

31. Holding, D. R. et al. The maize flouryl gene encodes a novel endoplasmic reticulum protein involved in zein protein body formation. Plant Cell 19, 2569-2582 (2007).

32. Clore, A. M., Dannenhoffer, J. M. \& Larkins, B. A. EF-1[alpha] is associated with a cytoskeletal network surrounding protein bodies in maize endosperm cells. Plant Cell 8, 2003-2014 (1996).

33. Saleh, A. A., Marion, D. \& Gallant, D. J. Microstructure of mealy and vitreous wheat endosperms (Triticum durum L.) with special emphasis on location and polymorphic behaviour of lipids. Food Struct. 5, 131-140 (1986)

34. Dickinson, A. J. et al. beta-Cyclocitral is a conserved root growth regulator Proc. Natl Acad. Sci. USA 116, 10563-10567 (2019).

35. Gabrielska, J. \& Gruszecki, W. I. Zeaxanthin (dihydroxy-beta-carotene) but not beta-carotene rigidifies lipid membranes: a 1H-NMR study of carotenoid-egg phosphatidylcholine liposomes. Biochim. Biophys. Acta 1285, 167-174 (1996).

36. Gruszecki, W. I. \& Strzałka, K. Carotenoids as modulators of lipid membrane physical properties. Biochim. Biophys. Acta 1740, 108-115 (2005)

37. Popova A. V., Andreeva A. S. Chapter Eight - carotenoid-lipid interactions. In: Advances in Planar Lipid Bilayers and Liposomes (eds Iglič, A. \& Genova, J.) (Academic Press, 2013).
38. Widomska, J., Welc, R. \& Gruszecki, W. I. The effect of carotenoids on the concentration of singlet oxygen in lipid membranes. Biochim. Biophys. Acta 1861, 845-851 (2019)

39. Harjes, C. E. et al. Natural genetic variation in lycopene epsilon cyclase tapped for maize biofortification. Science 319, 330-333 (2008).

40. Wu, Y. \& Messing, J. RNA interference-mediated change in protein body morphology and seed opacity through loss of different zein proteins. Plant Physiol. 153, 337-347 (2010).

41. $\mathrm{Li}, \mathrm{C}$. et al. Long-read sequencing reveals genomic structural variations that underlie creation of quality protein maize. Nat. Commun. 11, 17 (2020).

42. Zheng, X. et al. Intra-kernel reallocation of proteins in maize depends on VP1mediated scutellum development and nutrient assimilation. Plant Cell 31, 2613-2635 (2019)

43. Deng, Y., Wang, J., Zhang, Z., Wu, Y. Transactivation of Sus1 and Sus2 by Opaque2 is an essential supplement to sucrose synthase-mediated endosperm filling in maize. Plant Biotechnol. J. 18, 1897-1907 (2020).

44. Xie, Y. et al. Ultrasound-assisted one-phase solvent extraction coupled with liquid chromatography-quadrupole time-of-flight mass spectrometry for efficient profiling of egg yolk lipids. Food Chem. 319, 126547 (2020).

45. Liu, H. et al. Gene duplication confers enhanced expression of $27-\mathrm{kDa}$ gamma-zein for endosperm modification in quality protein maize. Proc. Natl Acad. Sci. USA 113, 4964-4969 (2016).

\section{Acknowledgements}

We thank Zhiping Zhang and Jiqin Li (CAS Center for Excellence in Molecular Plant Sciences, CAS) for technical support. We thank Dr. Dean DellaPenna from Michigan State University for his critical reading of this article. This research was supported by the Ministry of Science and Technology of China (2016YFD0100500 to YW), the National Natural Science Foundation of China (31830063 and 31925030 to YW) and the Chinese Academy of Sciences (XDB27010201 to YW).

\section{Author contributions}

H.W., Y.H., Q.X., X.H., C.L., X.G., Q.W., X.X., Y.Z., J.W. and W.W. conducted the experiments. Y.W. supervised the project. H.W., Y.H., B.L. and Y.W. explained the data, and drafted and edited the manuscript.

\section{Competing interests}

The authors declare that there is no conflict of interests regarding the publication of this article.

\section{Additional information}

Supplementary information is available for this paper at https://doi.org/10.1038/s41467020-19196-9.

Correspondence and requests for materials should be addressed to Y.W.

Peer review information Nature Communications thanks Didier Marion, Donald McCarty, Eleanore Wurtzel and the other, anonymous, reviewer(s) for their contribution to the peer review of this work. Peer reviewer reports are available.

Reprints and permission information is available at http://www.nature.com/reprints

Publisher's note Springer Nature remains neutral with regard to jurisdictional claims in published maps and institutional affiliations.

Open Access This article is licensed under a Creative Commons Attribution 4.0 International License, which permits use, sharing, adaptation, distribution and reproduction in any medium or format, as long as you give appropriate credit to the original author(s) and the source, provide a link to the Creative Commons license, and indicate if changes were made. The images or other third party material in this article are included in the article's Creative Commons license, unless indicated otherwise in a credit line to the material. If material is not included in the article's Creative Commons license and your intended use is not permitted by statutory regulation or exceeds the permitted use, you will need to obtain permission directly from the copyright holder. To view a copy of this license, visit http://creativecommons.org/ licenses/by/4.0/

(C) The Author(s) 2020 